Eur J Health Econ 2004 · 49:317-323

DOI 10.1007/s10198-004-0243-4

Published online: 12. August 2004

(c) Springer Medizin Verlag 2004

Tugrul Temel

Agricultural Economics and Rural Policy Group, Wageningen University,

Wageningen, The Netherlands

\title{
A method for cross-sector priority setting
}

\section{Gaps and hypotheses in malaria research: Tanzania}

he literature is rich, offering a wide range of priority setting methods. These methods are mostly applied to identify the key theme(s) within a sector by either a single or multiple criteria, or optimization techniques. They deal with, for instance, setting priorities in agricultural research or in environmental research in an isolated manner. What about priorities concerning multiple sectors concurrently? What about sources, intermediaries, and users of information on the prioritized areas? What about cause-effect pathways between sources and users of this information? What about the connectedness in a multisector system of organizations operating under different jurisdictions?

This study introduces a new priority setting method that considers all these concerns. It is a method for identifying critical information gaps within a given theme, such as malaria control, which concurrently concerns organizations from multiple sectors. (The term "gap" is used to refer to an area that warrants better understanding; the term "pathway," a chain of interactions between organizations; and the term "information pathway" that the content of the interaction concerned is information exchange.) The method uses graph-theoretical concepts and principles of systems theory in characterizing the underlying cause-effect information structure of the system under investigation see [9] for the principles of the systems theory; see $[4,6]$ on the use of the graph-theoretical concepts. It would prove useful especially in addressing cross-cutting problems for which alternative solutions lie in the establishment of effective information flow between the relevant organizations from different sectors. The key premise of the method is that identifying cause-effect information pathways in a system is essential for policy interventions to bring about desirable changes.

The application of the method is illustrated in the context of malaria control in Tanzania, using the findings of a workshop held in October 2003 in Dar es Salaam [14]. This is a relevant application because malaria is a cross-sector problem, and solutions are strongly dependent on the nature of information flow among the organizations in the system [15]. With a multivoting scheme, the workshop determined five critical gaps and two critical pathways that warrant better understanding. Interesting applications of the method would be to study such diseases as SARS and HIV/ AIDS of which causes and effects have not been well documented in the literature.

The study is organized as follows. In the next section, different priority setting methods are introduced and compared with the method introduced. The method is then explained by applying it in a malaria workshop held in Dar es Salaam in 2003. Afterwards, ways are discussed to clearly identify gaps and construct testable hypotheses by using the method. A model is then sketched for empirical testing of the hypotheses constructed. In the final section, the study is concluded.

\section{Where the new method stands in the literature}

There are a large number of studies applying different priority setting methods (for an extensive review see $[1,7,11])$. Not all methods are suited for all situations, and the choice of method is critical to the successful conclusion of a priority-setting exercise. The methods can be classified into two groups: single-criterion and multiplecriteria methods. The congruence method ranks research alternatives according to the value of a single measure such as value of production, number of poor who benefit from the anticipated research outcomes. Cost/benefit analysis compares in monetary terms anticipated or realized costs and benefits of research alternatives. The economic surplus method refines cost/benefit analysis, measuring the economic (efficiency) returns to investment in research. This method shows to what extent research-induced reductions in production costs may reduce market prices, and thus change the distribution of benefits between consumers and/or producers of a commodity.

Introduction. Earlier versions of this study were prepared, while the author was working at the International Service for National Agricultural Research (ISNAR). 
Multiple-criteria methods, on the other hand, combine assessments of the alternative research activities on various criteria. In a typical "scoring approach", the assessment of the individual criteria is based on subjective information, which is expressed in values on a numerical scale (e.g., from 1 to 9). Analytic hierarchy process is a more rigorous version of scoring methods. It is a multiobjective, multicriteria decisionmaking tool that employs pairwise comparisons to rank alternative solutions to a problem, formulated in hierarchic terms. The incorporation of all relevant decision criteria, and their pairwise comparison, allows decision makers to determine the trade-offs among objectives. Lastly, the mathematical programming method maximizes an objective function under constraints. Here only a selected number of methods are discussed to the extent that they relate to our method. (For further reading on the methods not discussed in this study, the reader is referred to the studies listed in the references.)

The application of our method involves three steps. In Step 1 sectors related to the problem at hand are organized as a system. This system constitutes components, each of which includes organizations that share a comparable objective and operate under similar resource constraints. With its own objective and resource constraints, the system itself represents a unified body that introduces at least one new property, which components cannot support individually. This would imply that the system is greater than the sum of its components. In Step 2 the information flowing in the system is adjusted by redefining that information on the basis of its ultimate influence on the components concerned. This adjustment helps identify cause-effect information pathways. In Step 3, by using causeeffect coordinates, dominant and subordinate components and their relative positions are detected. This is the step where research gaps and hypotheses to be tested are developed.

Our method differs in some ways from the methods mentioned above and resembles in some others. The key difference is that it adopts a systems approach to address a cross-sector disease control. It has been designed in such a way to identify priorities within a given theme that relates to organizations from multiple sectors. Cause-effect pathways among the priorities represent empirically testable hypotheses that can also be used in the design of policy interventions. The proposed method is a mixture of mathematical programming and scoring methods. It is similar to the mathematical programming method, as the system at hand is organized around an optimization problem, with a system goal maximized under system and sector specific constraints. This problem assumes that there is a benevolent body behind the optimization, and that all the sectors involved cooperate to optimize a common goal. It is also similar to scoring method, as binary causal relations in the system are prioritized subjectively as outcomes of a voting scheme. Once the priorities are set, the conventional methods, such as cost/benefit, economic surplus, and mathematical programming, can be applied to rank the cause-effect pathways concerned.

\section{The method}

This section describes the proposed method and illustrates its application in the context of a cross-sector workshop on malaria control in Tanzania. Malaria is multifactorial in its cause and cross-sectoral in its solution. Conventionally, the epidemiology of malaria concerns factors relating to the nature and spread of the Plasmodium parasite and its vectors, mosquitoes, the human host, and their interaction within a natural environment $[3,8]$. More recently, however, close association of malaria prevalence with poverty has led to research on the effect of economic, social, and political factors on the epidemiological triad agent, host, and environment $[5,10,12,13]$. Another critical association documented in the literature is with agriculture $[14,15$, 16]. These factors originate from different sectors, and hence controlling them simultaneously requires cross-sector collaboration. The following definition of a malaria control system describes the structure of this collaboration.

\section{Malaria control system S}

S is a set of organizations that jointly and/ or individually contribute to the genera- tion, dissemination, and use of existing or new information that directly and/or indirectly improves living conditions through reduced malaria. In $\mathrm{S}$, highly variable and context-sensitive information flows, the meaning and the value of which depend on the competencies of the interacting organizations [18].

\section{Components of S}

$S$ is described as a system of five components, $\{\mathrm{H}, \mathrm{V}, \mathrm{E}, \mathrm{A}, \mathrm{P}\}$, placed in the diagonal cells of S. The "human health" component $\mathrm{H}$ regards human beings as hosts to malaria mosquitoes. It should ideally comprise all the health organizations combating malaria. Among them organizations are ministry of health, hospitals, research and diagnostic laboratories, health information centers, health research institutes, medical supply firms, nongovernmental organizations (NGOs), and donors.

The "vector" V regards Plasmodium ppecies as causal agents, and mosquitoes, as vectors that bridge the gap between malaria and human beings. It includes such organizations dealing with parasite and vector ecology, such as changes in vector density, distribution, feeding habits, and prevalence. Research and diagnostic laboratories, research institutes, NGOs, and donors are some of the relevant organizations.

The "environment" E represents physical surrounding of human beings and mosquitoes. Thus all the organizations aimed at improving the quality of environmental resources should be included in this component. Examples include ministry of environment, meteorology centers, environmental research institutes, land cadastral units, water associations, NGOs, and donors.

The "agriculture" A represents agricultural systems, agricultural technologies, and resource development and should include such organizations as ministry of agriculture, extension and information units, agricultural research institutes, farms, agroindustries, input quality control bodies, NGOs, and donors.

Lastly, the "socioeconomic" component $\mathrm{P}$ represents the social and economic dimensions of malaria. It includes economic and social organizations that directly or indirectly affect malaria through mone- 
tary and nonmonetary resource allocation. Such organizations are ministry of economy, ministry of finance, ministry of cultural affairs, grass root organizations, NGOs, international organizations such as the World Bank, and municipalities:

$S=\left[\begin{array}{ccccc}H & H V & H E & H A & H P \\ V H & V & V E & V A & V P \\ E H & E V & E & E A & E P \\ A H & A V & A E & A & A P \\ P H & P V & P E & P A & P\end{array}\right]$

\section{Causal relations between the components}

Causal relations between the five components are placed in the off-diagonal cells of S. Following clockwise convention, each offdiagonal cell represents a relation between two components. For illustrative purposes, a small set of factors is considered. HV in $\mathrm{S}$ denotes " $\mathrm{H}$ causes V," indicating "information on effects of health interventions on mosquito density." Similarly, VH denotes "V causes H," indicating "information on effects of mosquito density on health interventions." Binary causal relations in the first row of S therefore indicate how $\mathrm{H}$ (i.e., human health-related organizations) is assumed to affect organizations in the rest of the system by either generating or disseminating the information concerned, and those in the first column indicate how organizations in the rest of the system are assumed to affect $\mathrm{H}$ by generating or disseminating the respective information.

$S$ is further characterized by pathways of binary causal relations. For example, a three-edged pathway, EVHA, is one from $\mathrm{E}$ to $\mathrm{V}$ and then from $\mathrm{V}$ to $\mathrm{H}$ and then from $\mathrm{H}$ to $\mathrm{A}$. This pathway will be realized only if the relevant organizations collaborate during the process of generating, disseminating, and using the information concerned. The cell corresponding to EV, for example, indicates that $\mathrm{E}$ is to generate and disseminate information on the specificities of climate change expected, while $\mathrm{V}$ is to use this information to study expected changes in the distribution and types of mosquitoes. The cell corresponding to $\mathrm{VH}$ similarly shows that $\mathrm{V}$ is to generate and disseminate information on the specificities of mosquito density predicted, while
$\mathrm{H}$ is to use this information in the design of proper health interventions. Finally, the cell $\mathrm{HA}$ indicates that $\mathrm{H}$ is to generate and disseminate information on the specificities of the designed health interventions, while $\mathrm{A}$ is to use this information to study expected changes in farm productivity. Needless to say, an effective flow of information can only be realized if the organizations concerned have formal and/or informal linkages between them.

The total number of $k$-edged pathways in $S$ is calculated by $[n ! /(n-k-1) !]$, where $n$ and $k$ stand for the number of components and the number of edges concerned, respectively. The exclamation sign denotes factorial in statistical terms. Applying this formula, one can calculate, for instance, the number of two-edged pathways in $S$ by $[n ! /(n-k-1) !]=[5 ! /(5-2-1) !]=60$.

\section{Application of the method to malaria control}

A workshop or a questionnaire can serve as a means to gather the information necessary for identifying top priority causal relations in S. For illustrative purposes, analysis in the rest of this study draws on the findings of a workshop, organized in Dar es Salaam on 27-28 October 2003 , on malaria information needs, sources, and use in Tanzania [14]. This workshop gathered policy makers, researcher managers, scientists, and field experts from the health, ecology, environment, agriculture, NGO, socioeconomic, and political sectors to identify (a) critical areas in malaria research that warrant better understanding and (b) capacities necessary for addressing the critical areas. In this study we make use of (a) only.

The workshop was organized in three working groups, each with six participants from different sectors. Each participant had three votes: a "strong" vote worth 3 points, a "mediocre" vote worth 2 points, and a "weak" vote worth 1 point. This multivoting scheme aimed to allow the participants to rank their preferences over binary causal relations. By using $\mathrm{S}$, each working group prepared a map of causal relations that the group thought to be critical. The resulting three maps were then consolidated, and the participants voted over the causal relations in the consolidated map. The top three causal relations were then
Eur J Health Econ 2004 - 49:317-323

DOI 10.1007/s10198-004-0243-4

C) Springer Medizin Verlag 2004

Tugrul Temel

\section{A method for cross-sector priority setting. Gaps and hypotheses in malaria research: Tanzania}

\begin{abstract}
This study proposes a new priority setting method for identifying critical information gaps in a multisector system. The method uses graph-theoretical concepts and principles of systems theory in characterizing the underlying information structure of the system. Its application is illustrated in the context of malaria control in Tanzania, drawing on the findings of a workshop held in October 2003 in Dar es Salaam. The workshop identified two critical pathways, EVHA and EVHPA, which warrant better understanding. The first suggests that the malaria research should generate information on the effects of environmental changes $(E)$ on vector ecology $(V)$ and then on the effects of $V$ on human health $(H)$ and then on the effects of $\mathrm{H}$ on agriculture (A). Interpreted likewise, the second pathway additionally points to the need for information on the effects of socioeconomic conditions (P) on A.
\end{abstract}

\section{Keywords}

Cross-sector priority setting . Systems and graph theories.

Disease control · Malaria · Tanzania 
Table 1

Cause - effect (C, E) coordinates

\begin{tabular}{|lllll}
\hline & $S_{s}$ & $S_{M}$ & $S_{W}$ & $S_{T}$ \\
\hline$H$ & $(21,15)$ & $(14,8)$ & $(6,6)$ & $(41,29)$ \\
\hline V & $(9,24)$ & $(4,8)$ & $(4,2)$ & $(17,34)$ \\
\hline E & $(15,3)$ & $(6,2)$ & $(0,4)$ & $(21,9)$ \\
\hline A & $(9,24)$ & $(6,6)$ & $(4,1)$ & $(19,31)$ \\
\hline P & $(15,3)$ & $(2,8)$ & $(3,4)$ & $(20,15)$ \\
\hline
\end{tabular}

discussed in the working groups to identify capacities required to address the top three relations.

$\mathrm{S}_{\mathrm{s}}$ indicates the causal relations that received strong votes only, putting first things first. For instance [placed in the third row - second column of $S_{\text {s }}$, the relationship EV received 5 strong votes, which amounts to 15 points. With 15 points, EV stands out as the top priority causal relationship to be investigated, followed by $\mathrm{HA}$ and PA with 12 points each]:

$\mathbf{S}_{s}=\left[\begin{array}{ccccc}H & 3 & 3 & 12 & 3 \\ 9 & V & 0 & 0 & 0 \\ 0 & 15 & E & 0 & 0 \\ 3 & 6 & 0 & A & 0 \\ 3 & 0 & 0 & 12 & P\end{array}\right]$

$\mathrm{S}_{\mathrm{M}}$ indicates the causal relations that received mediocre votes only. With 6 points in the first row - fifth column of $S_{M}$, HP is the strongest causal relationship, followed by $\mathrm{HV}, \mathrm{VH}, \mathrm{EV}$, and $\mathrm{AH}$ with 4 points each:

$$
\mathbf{S}_{M}=\left[\begin{array}{ccccc}
H & 4 & 2 & 2 & 6 \\
4 & V & 0 & 0 & 0 \\
0 & 4 & E & 2 & 0 \\
4 & 0 & 0 & A & 2 \\
0 & 0 & 0 & 2 & P
\end{array}\right]
$$

$\mathrm{S}_{\mathrm{W}}$ indicates the causal relations that received weak votes only. With 4 points, $\mathrm{VH}$ is the strongest relationship, followed by $\mathrm{HP}$ and $\mathrm{AE}$ with 3 points each:

$$
\mathbf{S}_{W}=\left[\begin{array}{ccccc}
H & 2 & 1 & 0 & 3 \\
4 & V & 0 & 0 & 0 \\
0 & 0 & E & 0 & 0 \\
0 & 0 & 3 & A & 1 \\
2 & 0 & 0 & 1 & P
\end{array}\right]
$$

Finally, $\mathrm{S}_{\mathrm{T}}$ indicates the aggregate votes calculated as $\left(\mathrm{S}_{\mathrm{s}}+\mathrm{S}_{\mathrm{M}}+\mathrm{S}_{\mathrm{W}}\right)$. With 19 points, $\mathrm{EV}$ stands out as the top priority relationship, followed by VH with 17, PA with 15, HA with 14 points, and HP with 12 points (see [9] for studying the features of $S_{\mathrm{T}}$ ):

$$
\mathbf{S}_{T}=\left[\begin{array}{ccccc}
H & 9 & 6 & 14 & 12 \\
17 & V & 0 & 0 & 0 \\
0 & 19 & E & 2 & 0 \\
7 & 6 & 3 & A & 3 \\
5 & 0 & 0 & 15 & P
\end{array}\right]
$$

\section{Cause-effect structure of $S_{i}$ ( $\mathrm{i}=\mathrm{S}, \mathrm{M}, \mathrm{W}, \mathrm{T}$ )}

Cause (C) of a component in $\mathrm{S}$ is defined as the sum of the points in the corresponding row; and effect (E), as the sum of the points in the corresponding column of - Table 1. Mapping the (C,E) coordinates in - Table 1, $\bullet$ Figs. 1, 2, 3, 4 show the underlying structures of $S_{T}, S_{s}, S_{M}$, and $S_{W}$, respectively. These figures have three critical regions. Region 1 is the locus of the $45^{\circ}$ line, where $\mathrm{C}=\mathrm{E}$. A component on this line is said to be highly interactive with the rest of the system if its coordinate falls in the northeast corner of the figure; and minimally interactive if its coordinate is close by the $(0,0)$ coordinate. Region 2 is the area below the $45^{\circ}$ line, where $\mathrm{C}>\mathrm{E}$. A component with a very high $\mathrm{C}$ and a very low $\mathrm{E}$, denoted by $\mathrm{C}>>\mathrm{E}$, suggests that it strongly dominates over the others in the system. Region 3 is the area above the $45^{\circ}$ line, where $\mathrm{C}<\mathrm{E}$. A component with a very low $\mathrm{C}$ and a very high $\mathrm{E}$, denoted by $\mathrm{C}<<\mathrm{E}$, suggests that it is strongly subordinate.

The C-E structure of $S_{T}$ shown in - Fig. 1 reveals: (a) $\mathrm{H}$ is the most dominant component in the system, with a cause of 41 points, followed by $E$ with 21 points; (b) $\mathrm{P}$ is relatively speaking the most interactive component; and (c) V and A are both subordinate components. The observation (a) and the three key relations HA, HP, and $\mathrm{EV}$ in $\mathrm{S}_{\mathrm{T}}$ all together suggest that research needs to be carried out to uncover the mechanisms through which $\mathrm{H}$ influences both $\mathrm{A}$ and $\mathrm{P}$, and $\mathrm{E}$ influences V. Further-

\footnotetext{
1 I thank Rick Davies, in a personal communication, for indicating the usefulness of such figures; for visual graph-theoretic methods see [4].
}

more, the observation (b) and the key relationship PA in $\mathrm{S}_{\mathrm{T}}$ together suggest that research needs to be carried out to uncover the mechanisms through which P influences $\mathrm{A}$. Finally, that $\mathrm{H}$ is also influenced strongly by the rest of the system points to the need for further research as to how $\mathrm{V}$ influences $\mathrm{H}$. It should be noted that these three suggestions imply the following reduced form of $\mathrm{S}_{\mathrm{T}}$, indicating the identified critical relationships only.

$\mathbf{S}_{T}=\left[\begin{array}{ccccc}H & & & 14 & 12 \\ 17 & V & & & \\ & 19 & E & & \\ & & & A & \\ & & & 15 & P\end{array}\right]$

The reduced form of $S_{T}$ underlines the key feature of the system at hand. Environment (E) is the only truly exogenous component, while agriculture (A) is the only truly endogenous component. One implication of this feature is that pathways of interest in the reduced $S_{T}$ would always start with $\mathrm{E}$ and end at $\mathrm{A}$, including EVHA and EVHPA. All in all, the analysis suggests that pathways including EVHA and EVHPA warrant better understanding.

The C-E structure of $S_{s}$ shown in

- Fig. 2 is interpreted similarly. It shows an almost identical structure to that in - Fig. 1, except that the components are more polarized. Furthermore, the reduced form of $\mathrm{S}_{\mathrm{s}}$ has two exogenous components, $\mathrm{E}$ and $\mathrm{P}$, while A still remains to be the only endogenous component. Therefore the pathways of interest would include EVHA and PA. (We leave the interpretation of $S_{M}$ and $S_{W}$ to the reader.)

$\mathbf{S}_{S}=\left[\begin{array}{ccccc}H & & & 12 & \\ 9 & V & & & \\ & 15 & E & & \\ & & & A & \\ & & & 12 & P\end{array}\right]$

\section{Developing testable hypotheses}

The findings can be analyzed with three concepts: critical information gaps, critical cause-effect information pathways, and critical hypotheses. The multivoting scheme carried out resulted in the identifi- 
cation of five critical gaps that warrant better understanding (see the reduced forms of $S_{T}$ and $S_{s}$ and Figs. 1-2). These gaps are: the effects of environmental changes (E) on vector ecology $(\mathrm{V})$; the effects of changing vector ecology $(\mathrm{V})$ on human health $(\mathrm{H})$; the effects of changing human health $(\mathrm{H})$ on agricultural systems $(\mathrm{A})$; the effects of changing human health $(\mathrm{H})$ on socioeconomic conditions $(\mathrm{P})$; and the effects of changing socioeconomic conditions $(\mathrm{P})$ on agricultural systems (A). Each one of the gaps represents a hypothesis to be tested empirically.

The gaps further imply that the malaria control system under investigation has a total of two cause-effect information pathways, EVHA and EVHPA, to be examined. The first pathway, EVHA, should read as follows. The "environment" organizations should generate information on the effects of climate change, deforestation, water use, and environmental management practices on the malaria situation, and this information should be communicated with the "vector" organizations. The "vector" organizations would then use this information in its research or field activities and pass it onto the "human health" organizations for expected use in the design of public health interventions. Finally, the "health" organizations should communicate the possible effects of health interventions on the agricultural productivity. Interpreted likewise, the second pathway additionally indicates the need for information on the effects of changing health status on socioeconomic conditions and the effects of changing socioeconomic conditions on agricultural system. Note that the sequence of interactions in these pathways is crucial and remains to be tested.

The directed causal relations in Figs. 1 and 2 show that $\mathrm{H}$ is the dominant source of information, which is followed by $\mathrm{E}$ and $\mathrm{P}$, and that $\mathrm{V}$ and $\mathrm{A}$ are the subordinate users of information. Each one of these observations represents an area to be investigated empirically. Regarding $\mathrm{H}$ being the dominant source, one can formulate a hypothesis of whether public health interventions significantly influence agricultural productivity or malaria transmission. Likewise, the influence on $\mathrm{A}$ and $\mathrm{V}$ of the second degree sources (E and P) can also be tested.

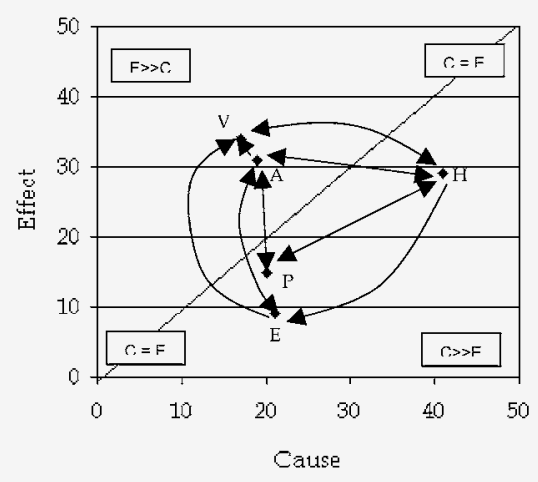

Fig. $1 \Delta$ Cause - effect structure of $S_{T}$

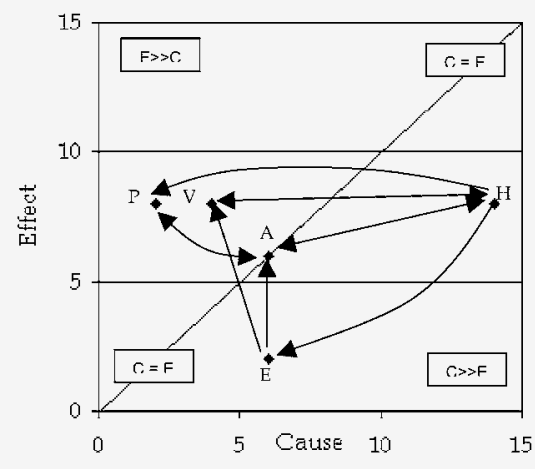

Fig. $3 \Delta$ Cause - effect structure of $S_{M}$

The analysis indicates that some components have distinct testable characteristics. The first characteristic is that $\mathrm{E}$ is exogenous, generating information without input from other components, or that participants view $\mathrm{EV}$ as one of the most important information gaps in the malaria control system. This immediately follows from the reduced forms of $S_{T}$ and $S_{s}$, where the column associated with $\mathrm{E}$ is empty. The second is that A is endogenous, absorbing information but not letting it go. This follows from the reduced forms of $S_{T}$ and $S_{s}$, where the row associated with $\mathrm{A}$ is empty.

The multivoting scheme was applied to classify binary relations into three groups: high, mediocre, and weak. The implied information structures are then mapped in Figs. 2, 3, and 4, respectively. A comparison of these structures points to two regularities to be investigated further. First, no matter which group is used, $\mathrm{H}$ remains to be the most crucial source of information. Second, A shows the highest variability on the spectrum: subordinate in $\bullet$ Fig. 2 , interactive in $\bullet$ Fig. 3, and dominant in • Fig. 4.

The discussion in this section draws only on the findings of the specific workshop held in Tanzania and therefore should not

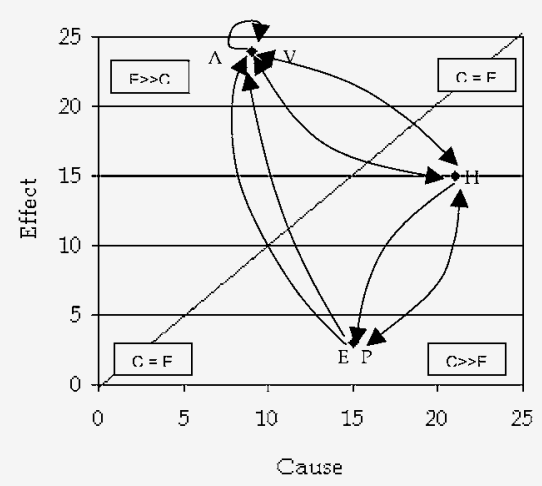

Fig $2 \Delta$ Cause - effect structure of $\mathrm{S}_{\mathrm{s}}$

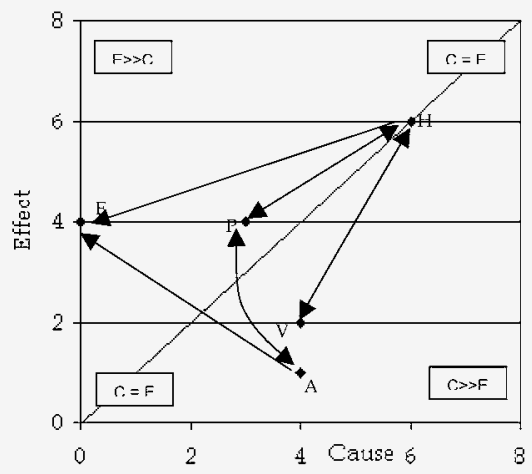

Fig. $4 \Delta$ Cause - effect structure of $S_{w}$

be generalized to the entire country. For a representative analysis of critical hypotheses to be investigated in the context of malaria in Tanzania, the method should be applied to map causal relations that have been already studied in the literature. Such a literature mapping would also allow us to identify the true critical gaps that remain unaddressed.

\section{A structure for information system}

Top priorities in malaria research were identified by using expert knowledge; however, the measurement of "marginal effects" (i.e., parameters that correspond to the off-diagonal cells of S) remains to be determined whether one wishes to rank the very same priorities with respect to their economic benefits to the community concerned. In this section we sketch a model that one can use to estimate these parameters.

Suppose that component $i \in\{H, V, E, A$, $P\}$ either produces information or transmits information from one component to another or uses information transmitted by others or performs all these functions. Component is information man- 
agement technology can be specified as follows ("management technology" refers to three functions simultaneously: information production, transmission, and use:

$I_{i, t}=T_{t} F_{i}\left(I_{i, t-1}, L_{i, t}, D_{i, t} ; I_{-i, t-1}\right)$

with $T_{t}=T\left(D_{t}, L_{t}\right)$

where:

- $\mathrm{I}_{\mathrm{i}, \mathrm{t}}$ : The stock of information produced by all the organizations in the $i$ th-component in period $t . \mathrm{I}_{\mathrm{i}, \mathrm{t}-\mathrm{1}}$ is the prior stock of information.

- $\mathrm{L}_{\mathrm{i}, \mathrm{t}}$ : The learning capacity of the $i$ thcomponent in period $t$, which is the average capacity of organizations in that component.

- $\mathrm{D}_{\mathrm{i}, \mathrm{t}}$ : The density (or connectedness) of the $i$ th-component in period $t$.

- $\mathrm{I}_{-\mathrm{i}, \mathrm{t}-\mathrm{1}}$ : The stock of information produced in period $t-1$ by all but the $i$ th component. The subscript $-i$ indicates that the $i$ th-component is excluded.

- $\mathrm{T}_{\mathrm{t}}$ : System technology in period $t$, over which individual organizations have no influence.

- $\mathrm{D}_{\mathrm{t}}$ : System density (or connectedness of all the components) in period $t$.

- $\mathrm{L}_{\mathrm{t}}$ : System learning capacity in period $t$.

The system density, $D_{t}$, is calculated as $b /[n(n-1)]$ with $1 \geq \mathrm{D}_{t} \geq 0$, where $b$ is the total number of the identified causal relations, and $n$ is the number of the dimensions of the system. For example, the density for the aggregate system $\mathrm{S}_{\mathrm{T}}$ is $(13 / 20)$, where $b=13$ and $n=5$. The density of component $i$ is calculated as $b_{i} / 2(n-1)$, where $b_{i}$ is the number of component $i$ 's identified causal relations. For example, from $\mathrm{S}_{\mathrm{T}}$, component $i$ has a density of ( $7 / 8)$, where $b=7$.

This specification assumes that component $i$ can improve its performance through investing in component-specific learning $\left(L_{i, t}\right)$ activities and activities that enhance its degree of connectivity $\left(D_{i, t}\right)$ with others in the system ${ }^{2}$. Learning

2 Theodorakopoulou and Kalaitzandonakes [17] introduce a similar model except that our model of information production additionally considers the system characteristics represented by $T$ (.) and other components' existing stock of information denoted by $I_{-i, t-1}$, which are both exogenous to individual organizations' information production decisions. and connectivity represent the two decision variables of component $i$. The vector $I_{-i, t-1}$ is exogenous to component $i$, which is, for instance, equal to $\left(I_{V, t-1}, I_{E, t-1}, I_{A, t-1}\right.$, $\left.I_{P, t-1}\right)$ if $i=\mathrm{H}$. $T(.,$.$) represents an exoge-$ nous process that influences the effectiveness of all the components in the system; it only changes with time. This is the process that takes into account the external effects of the system itself on individual components. The components are connected to each other through $I_{-i, t-1}$ and are all subject to the same external effects.

A system information management technology, representing a system goal, $S_{t}$, to which all the components contribute (the system goal is purposefully denoted by $S_{t}$, which is also used in the definition of a malaria control system) can be specified as:

$S_{t}=T_{t} F_{S}\left(I_{\bullet i, t}, M_{t}\right)$

where $I_{\cdot i, t-1} \equiv\left(I_{H, t} I_{V, t} I_{E, t} I_{A, t} I_{P, t}\right)$. The system management variable, $M_{t}$, captures positive effects on the system goal of change and linkage management. The task is to be performed by a benevolent body, as the benefits are pure public goods. This new variable would capture one new property, which components cannot support individually. This would imply that the system is greater than the sum of its components. In our context, $S_{t}$ refers to improved living conditions through reduced malaria. Given a level of $S_{t}$, one can derive marginal effects, which are represented by binary cause-effect relations placed in the off-diagonal cells of $S$, from the system information management technology.

\section{Conclusions}

This study proposes a new priority setting method for identifying critical information gaps in a multisector system. This method uses graph-theoretical concepts and principles of systems theory in characterizing the underlying cause-effect information structure of the system under investigation. The application of the method is illustrated in the context of malaria control in Tanzania, drawing on the findings of a workshop held in October 2003 in Dar es Salaam.
The workshop identified five critical information gaps (EV, VH, HA, HP, PA) and two cause-effect information pathways (EVHA and EVHPA) that warrant better understanding. These pathways imply that time has come to go beyond the epidemiological triad pathway $(\mathrm{EVH})$ and investigate the role that $\mathrm{A}$ and $\mathrm{P}$ are likely to play in the malaria transmission in Tanzania. Furthermore, a comparison of the three implied information structures points to two regularities to be investigated further. First, no matter which group is used, $\mathrm{H}$ remains to be the most crucial source of information. Second, A shows the highest variability in the system. All these results remain to be tested empirically.

On the methodological account, the method proposed can be developed further in three areas. First, the system under examination is represented by matrix $S$, where the flow of information is presented in an input-output matrix format. (Leontief's input-output matrix is widely used in economic modeling, which shows the flow of resources and final outputs among economic actors; the matrix $\mathrm{S}$ has been constructed in the same spirit as in Leontief's.) As opposed to Leontief's matrix, however, $S$ does not allow loops between those organizations that belong to the same component and hence does not give full account of information flow in the system.

Second, the information flow in the system must be standardized for comparability of the effects of pathways identified. A common unit must be used for measurement; however, this is difficult because the value of information depends on the specific situation of the involving parties [2]. The organizations interact with each other during the process of the generation, exchange, and use of the relevant information. The sole content of this interaction is information that directly and/or indirectly concerns malaria control. The value of the information would determine the speed and extent of information flow in S. The systems approach introduced by the current study implicitly assumes that there is a benevolent decision-making body whose only goal is to improve living conditions by reduced malaria incidence, given the system constraints. (The reader is also referred to the public economics lit- 
erature for further reading on the value of public goods.)

Third, developing an information accounting system requires a clear-cut distinction between formal and informal information. As Wolf et al. [18] have argued, the distinction lies in the communication medium and the intentions underlying specific interpersonal contact. Formal information is defined as being derived through structured channels generally in the form of text but also including conferences, phone calls, and other forms organized for the explicit purpose of information exchange. Conversations and social interactions among family, friends, and business associates including colleagues, customers, suppliers, and competitors constitute informal information. Of course, the distinction between social interaction and explicit information exchange is not clearcut as personal, family, community, and economic spheres overlap.

On the empirical account, several weaknesses exist that relate to the quality of the findings of the workshop and hence the quality of the results claimed by this study. First, for a consistency check, the mapping of the gaps identified in the workshop should be compared with that implied by the literature survey on malaria in Tanzania. The literature mapping is left for future research, as the main objective of this study is to introduce the priority setting method concerned. A second weakness, which is related to the first one, is that at this moment one cannot assess whether the participants made a distinction between critical gaps that warrant better understanding and critical areas they are aware that enough research has been carried out so far. This weakness could have been avoided though by conducting two mapping exercises in the workshop: one for critical gaps the participants are aware that enough research has been conducted and another for critical gaps that warrant further research. A third weakness is that some important gaps, for example, effects of agricultural activities on malaria prevalence and effects of endogenous environmental deterioration on malaria, were simply not recognized. This can partly be attributed to the limited knowledge of the participants and partly to the composition of working groups in which some sectors are under or overrepresented.

\section{Corresponding author Tugrul Temel}

Agricultural Economics and Rural Policy Group, Wageningen University, The Netherlands e-mail: tugrul.temel@wur.nl

\section{Acknowledgements}

I thank each of the participants at the Dar es Salaam workshop for providing insights into the dynamics of malaria in Tanzania. I also thank Anna Wuyts, Ninatubu Lema, and Barnabas Kapange for the organization of this workshop; Michael Loevinsohn for his comments on the design of the workshop; and Kayo Narita for preparation of the manuscript. I also acknowledge the financial support from Systemwide Initiative on Malaria and Agriculture (SIMA) and ISNAR for the organization of the workshop. This paper draws on ISNAR briefing paper no. 75.

\section{References}

1. Alston JM, Norton G, Pardey P (1995) Science under scarcity: principles and practice for agricultural research evaluation and priority setting. Ithaca: Cornell University Press

2. Arrow JK (1986) The value of and demand for information. In: McGuire CB, Radner R (eds), Decision and organization, 2nd edn. Minnesota: University of Minnesota Press

3. Clyde DF (1987) Recent trends in the epidemiology and control of malaria. Epidemiol Rev 9:21-243

4. Freeman LC (2000) Using available graph theoretic or molecular modeling programs in social network analysis (unpublished)

5. Gallup JL, Sachs JD (2001) The economic burden of malaria. Am J Trop Med Hyg 64:85-96

6. Hudson JA (1992) Rock engineering systems: theory and practice. London: Horwood

7. Janssen W (1995) Priority setting as a practical tool for research management. In: Bosch M, Preuss H$J A$ (eds) Management issues in national agricultural research systems: concepts, instruments, experiences. Hamburg: LIT

8. Krier JP, Baker JR (eds) (1980) Parasitic protozoa, vol 7, 2nd edn. New York, Academic

9. Murota K (1987) Systems analysis by graphs and matroids: structural solvability and controlability. Springer, Berlin Heidelberg New York

10. Panvisavas $S$ (2001) Poverty and malaria: a study in a Thai-Myanmar border area. Southeast Asian J Trop Med Public Health 32:608-614

11. Saaty T, Vargas L (1994) Decision making in economic, political, social and technological environments with the AHP. Pittsburgh: University of Pittsburgh

12. Sachs J, Malaney P (2002) The economic and social burden of malaria. Nature 416:581

13. Sharma SK, Pradhan P, Padhi DM (2001) Socio-economic factors associated with malaria in a tribal area of Orissa. Indian J Public Health 45:93-98
14. Temel T (2003) Proceedings of workshop: Tanzania. A joint ISNAR-SIMA Report. The Hague, Netherlands: ISNAR

15. Temel T, Maru A (2004) A systems approach to malaria control: institutional perspective. Forthcoming in Health Policy

16. Temel $T$ (2004) Malaria from the gap: need for cross-sectoral cooperation. Acta Tropica 89:249259

17. Theodorakopoulou I, Kalaitzandonakes N (2001) Structure of public-private knowledge networks in plant biotechnology: an EU-US comparison. In: Wolf S, Zilberman D (eds) Knowledge generation and technical change: institutional innovation in agriculture. Boston: Kluwer

18. Wolf S, Zilberman D, Wu S, Just D (2001) Institutional relations in agricultural information systems. In: Wolf S, Zilberman D (eds) Knowledge generation and technical change: institutional innovation in agriculture. Boston: Kluwer 